\title{
Dielectric constant boost in amorphous sesquioxides
}

\author{
Pietro Delugas, ${ }^{1,2}$ Vincenzo Fiorentini, ${ }^{1,3,4}$ and Alessio Filippetti ${ }^{3}$ \\ 1) NXP Semiconductors, Leuven, Belgium \\ 2) CNR-IMM, Catania, Italy \\ 3) CNR-INFM-SLACS, Cagliari, Italy \\ 4) Dipartimento di Fisica, Università di Cagliari, Italy
}

(Dated: October 29, 2018)

\begin{abstract}
High- $\kappa$ dielectrics for insulating layers are a current key ingredient of microelectronics. $\mathrm{X}_{2} \mathrm{O}_{3}$ sesquioxide compounds are among the candidates. Here we show for a typical material of this class, $\mathrm{Sc}_{2} \mathrm{O}_{3}$, that the relatively modest dielectric constant of its crystalline phase is enhanced in the amorphous phase by over $40 \%$ (from $\sim 15$ to $\sim 22$ ). This is due to the disorder-induced activation of low frequency cation-related modes which are inactive or inefficient in the crystal, and by the conservation of effective dynamical charges (a measure of atomic polarizability). The analysis employs density-functional energy-force and perturbation-theory calculations of the dielectric response of amorphous samples generated by pair-potential molecular dynamics.

PACS numbers: 77.22-d,63.20-e,78.30-j,61.66-f
\end{abstract}

High-dielectric constant ("high- $\kappa$ ") materials are gradually replacing silica [1] as gate dielectrics in integrated electronics. Policrystalline layers thereof usually behave badly in electrical terms due to grain-boundary conduction, so that amorphous layers are seriously studied as well. Whether or not the $\kappa$ of the crystal will be conserved upon amorphization is not obvious a priori [2], and should be assessed during the validation of a candidate dielectric to be used in thin insulating coatings.

Considerable attention has been devoted recently $[3,4]$ to sesquioxides $\mathrm{X}_{2} \mathrm{O}_{3}$ with the trivalent cation $\mathrm{X}=\mathrm{Y}, \mathrm{Sc}$, or a rare-earth atom. Almost all these materials adopt the cubic bixbyite crystal structure. Experiments [3, 4] and theory [4, 5] largely agree that their static dielectric constant in that phase is in the order of 12 to 15 , and of vibrational origin for about $\sim 60-70 \%$. The microscopic rationale for this behavior was given recently by the present authors [4, 5]: the infra-red (IR) active vibrational modes are relatively high-frequency and essentially cation-independent motions.

As we discussed in detail elsewhere [2, 6, , 7, 8], the dielectric constant $\kappa_{s}=\kappa_{\infty}+\kappa_{\text {ion }}$ in high- $\kappa$ materials is generally dominated by the lattice-vibrational component $\kappa_{\text {ion }}$, a factor 2 to 10 larger than the electronic $\kappa_{\infty}$. By its definition

$$
\kappa_{\text {ion }}^{\alpha \beta}=\frac{4 \pi e^{2}}{\Omega} \sum_{\lambda} \frac{z_{\lambda \alpha} z_{\lambda \beta}}{\omega_{\lambda}^{2}}, \quad z_{\lambda \beta}=\sum_{i \beta} \frac{Z_{i, \alpha \beta}^{*} \xi_{i, \lambda \beta}}{\sqrt{M_{i}}},
$$

(with $z$ the mode charge vector, $\Omega$ the system volume, $Z_{i, \alpha, \beta}^{*}$ the effective or dynamical or Born charge tensor and $M_{i}$ the mass of atom $i, \xi_{i, \lambda \beta}$ and $\omega$ the eigenvector and eigenfrequency of mode $\lambda$ at zero wave-vector, and $\alpha, \beta$ cartesian indexes), a large $\kappa_{\text {ion }}$ is due to large effective polarizabilities as measured by dynamical charges, termed "anomalous" if they exceed nominal ionicity, and to soft IR vibrational modes. A question that naturally arises is, will amorphization, i.e. microscopic dis- order, deteriorate the dielectric constant of these oxides ? In this Letter we show, based on ab initio calculations, that scandia, $\mathrm{Sc}_{2} \mathrm{O}_{3}$, has a larger dielectric constant in its amorphous phase than in its crystal phase. This dielectric constant boost is due to a) hardly any loss in dynamical polarizability, and b) disorder-induced IR activation of non-polar low-energy modes related to cation-oxygen combined motions. Previously [4, 5] we found that the vibrational dielectric behavior in cubic crystalline sesquioxides is dominated by oxygen motions and essentially independently of the cation. Therefore the present predictions for scandia are highly likely to apply to all sequioxides. Further, we point out that this behavior is not unique: recently, we have shown [2] that also $\mathrm{RAO}_{3}$ oxides ( $\mathrm{R}$ a rare-earth and $\mathrm{A}$ a trivalent cation) roughly conserve their dielectric constant in the amorphous phase.

Amorphous $\mathrm{Sc}_{2} \mathrm{O}_{3}$ samples were generated by a meltand-quench procedure using empirical-potential molecular dynamics as implemented in the GULP code [9]. A periodic 80-atom, cubic, crystalline $\mathrm{Sc}_{2} \mathrm{O}_{3}$ sample was molten, and the liquid was equilibrated at $\mathrm{T}=5000 \mathrm{~K}$ for $200 \mathrm{ps}$ to achieve fully randomized cation positions. It was then quenched down to $500 \mathrm{~K}$ by Nose' constant$\mathrm{T}$ dynamics at a cooling rate $20 \mathrm{~K} / \mathrm{ps}$, in 5-ps costant$\mathrm{T}$ intervals separated by abrupt $100 \mathrm{~K}$ down-steps. At $\mathrm{T}=500 \mathrm{~K}$, structural properties (well converged [10] at this cooling rate) of the amorphous sample were sampled in a 5 ps run. Tests on the cell size ( 80 to 320 atoms) did not indicate appreciable changes in the microscopic structure.

After bringing the system to $0 \mathrm{~K}$ by damped dynamics, the structure and volume were optimized (for the 80atom sample only) using the ab initio density-functional theory code Espresso [11]. Dynamical charges were computed on the final structure by density functional perturbation theory [12]. Phonon frequencies at zero wavevector were obtained from the force constant matrix calcu- 
lated by finite-difference force calculations (with cartesian displacements of \pm 0.01 bohr $)$. The technical details of the DFT calculation 11 are closely similar to those of our previous investigation on $\mathrm{RAO}_{3}$ perovskite-derived amorphous samples [2]. DFT calculations are indicated hencefort by the acronym GGA (i.e. generalized-gradient approximation [11]).
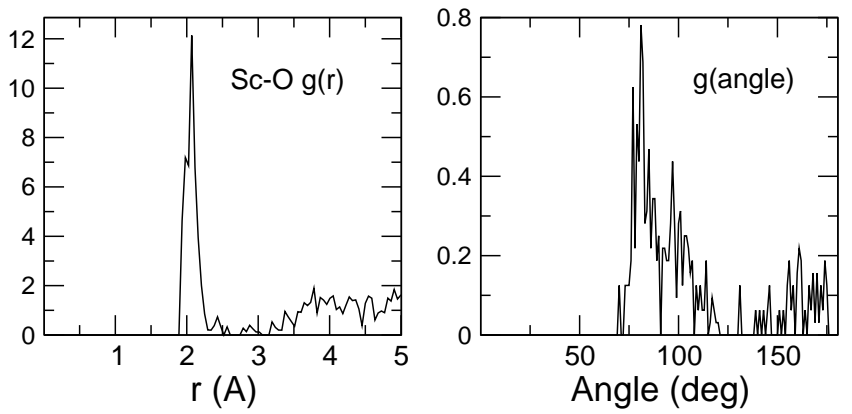

FIG. 1: Pair correlation functions for the Sc-O pair (left panel) and distribution of O-Sc-O bond angle (right panel) for $\mathrm{Sc}_{2} \mathrm{O}_{3}$.

In bixbyite sesquioxides, the cations are all six-fold coordinated, one out of four in the primitive cell has six oxygen neighbors at a single distance, the others have three pairs of neighboring O's at three distinct distances differing by about $0.1 \AA$. In amorphous scandia, as shown by Fig. 1, left panel, there is still a quite well defined Sc-O peak. Its width is about twice the crystal value. Correlations at larger distances are washed out by disorder. The same goes for the Sc-O bond angle distribution, which peaks at about $80^{\circ}$, shows an approximate bimodal structure (at about 80 and $100^{\circ}$ ), and is appreciably non-zero all the way from $70^{\circ}$ to $120^{\circ}$, indicating a strong orientational disorder or the octahedra making up the structure.

The dynamical polarizability of vibrating ions is correctly measured by the effective dynamical charge tensor $Z_{i, \alpha \beta}^{*}=\Omega / \mathrm{e}\left(\partial P_{\alpha} / \partial u_{\beta}\right)$, i.e. the polarization derivative with respect to atomic displacement 13]. A loss of effective polarizability will to decrease the ionic dielectric activity. The GGA dynamical charges of the amorphous and crystal phases in Table I are seen to be essentially the same. The amorphous-phase charge and dielectric tensors are averaged over all atoms in a given species and decomposed into $s, p$, and $d$ components [2]; Table [I reports the $s$ component, which contributes in excess of $98 \%$ of the tensor norm, is reported for the amorphous case.

The electronic dielectric constant is almost unaffected by disorder; the amorphous phase indeed has a crystallike gap in the electronic density of states, as expected from ionicity and from the anion (cation) character of valence (conduction) states. The static value in the amorphous phase is $40 \%$ larger than the crystal value. The ionic component is larger in the amorphous. To
TABLE I: Average $s$ components of the effective-charge tensor, and of the electronic, ionic and total static dielectric constant tensors in amorphous and bixbyite-crystalline $\mathrm{Sc}_{2} \mathrm{O}_{3}$. The slight difference in oxygen charges is related to differences in the (minor) violations of the dynamical charge neutrality sum rule.

\begin{tabular}{cccccc}
\hline \hline $\mathrm{Sc}_{2} \mathrm{O}_{3}$ & $\left\langle Z_{\mathrm{Sc}}^{*}\right\rangle$ & $\left\langle Z_{\mathrm{O}}^{*}\right\rangle$ & $\kappa_{\infty}$ & $\kappa_{\text {ionic }}$ & $\kappa_{s}$ \\
\hline crystal & 3.82 & -2.54 & 4.6 & 10.4 & 15 \\
amorphous & 3.82 & -2.53 & 4.5 & 17.5 & 22 \\
\hline \hline
\end{tabular}

understand why this occurs, we analyze the frequencydependent dielectric intensity, namely the dimensionless individual terms in the first equality of Eq.1. Before that, we note in passing that experiments have indeed shown a comparable enhancement: unfortunately, scandia recrystallizes during post-growth thermal treatments, so the enhancement effect is destroyed. Recrystallization occurs for most sesquioxides [14]; whenever it doesn't, an enhancement is indeed observed 15]

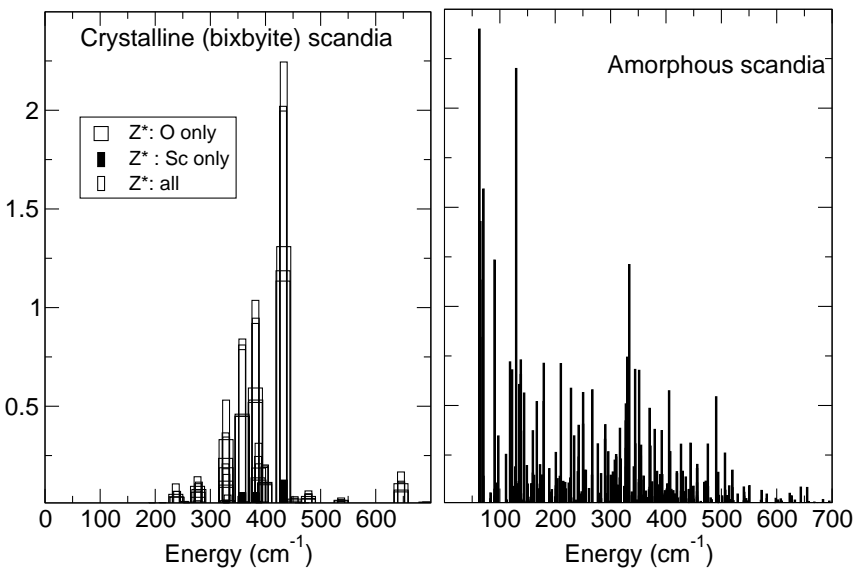

FIG. 2: Dielectric intensity for crystalline (bixbyite) scandia (left panel) and amorphous scandia (right).

In Fig 2 we compare the dielectric intensity of crystalline bixbyite-structure (right) and amorphous (left) $\mathrm{Sc}_{2} \mathrm{O}_{3}$. Visibly, in $\mathrm{Sc}_{2} \mathrm{O}_{3}$ as in other bixbyites the main IR modes fall around $300-400 \mathrm{~cm}^{-1}$. As can be appreciated from the decomposition, these modes are almost entirely oxygen-related; modes involving cation motions have negligible intensity at low energy, and small in the active IR range (similarly, despite the different intensity ratios, to e.g. $\mathrm{Lu}_{2} \mathrm{O}_{3}[4]$ ). In the amorphous phase, the dielectric enhancement at low frequency is due to IRinactive (generally Raman) or inefficient crystal modes that acquire IR character (or enhance it) due to disorder: as Eq.1 shows, the dielectric intensity at low frequency can be dramatically amplified due to a $1 / \omega^{2}$ factor provided a non-negligible intensity is present. These new modes are mainly Sc-related: this is evident from Fig 3, which reports the dipole amplitudes decomposed by atomic species (essentially the "charge vectors" $z_{\lambda}$ de- 
fined in Eq.1 obtained summing over one species only). Low frequency modes are seen to be dominated by Sc motions.

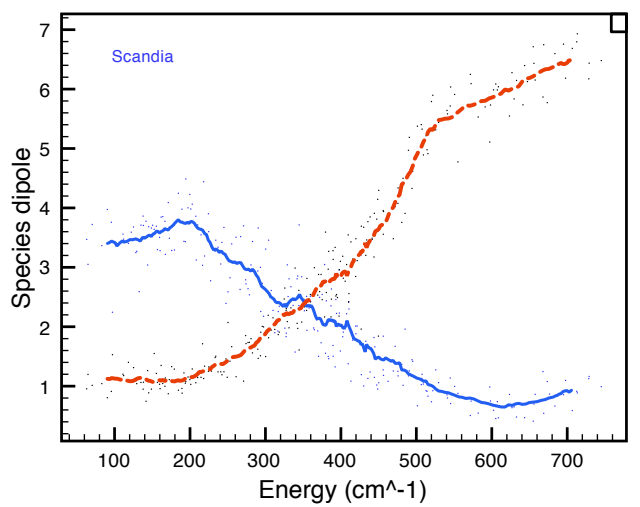

FIG. 3: Average atom-specific dipoles in amorphous scandia $\mathrm{Sc}_{2} \mathrm{O}_{3}$ (solid line: Sc; dashed: $\mathrm{O}$ ). Curves are obtained as window averaging (smoothing) of the data points shown as dots.

In summary, we have shown that the static dielectric response of amorphous scandia $\mathrm{Sc}_{2} \mathrm{O}_{3}$ is enhanced compared to that of its crystal phase, due to a combination of polarizability conservation (effective charges) and of disorder-induced IR activation of inefficient cationrelated modes. Whereas their crystal phase has poor dielectric constant $(\sim 15)$, we suggest that amorphous $\mathrm{X}_{2} \mathrm{O}_{3}$ materials can typically exhibit intermediate dielectricconstant values $(\sim 22)$ in the amorphous phase.

Work partially supported by EU (project FUNC@NXP Leuven), MiUR Italy under PON-Cybersar, Fondazione Banco di Sardegna. Most calculations were done on the SLACS cluster at CASPUR Rome.

[1] See MRS Bulletin vol. 27, n.3, Special Issue on Advanced Gate Dielectrics for Microelectronics.
[2] P. Delugas, V. Fiorentini, A. Filippetti, and G. Pourtois, Phys. Rev. B. 76, 104112 (2007).

[3] See e.g. R. D. Shannon, J. Appl. Phys. 73, 348 (1993); T. Busani and R. A. B. Devine, ibid. 98, 44102 (2005).

[4] E. Bonera, G. Scarel, M. Fanciulli, P. Delugas, and V. Fiorentini, Phys. Rev. Lett. 94, 027602 (2005).

[5] P. Delugas and V. Fiorentini, to be published.

[6] P. Delugas, V. Fiorentini, and A. Filippetti, Phys. Rev. B 71, 134302 (2005).

[7] P. Delugas, V. Fiorentini, and A. Filippetti, in Rare Earth Oxide Thin Films: Growth, Characterization and Applications, M. Fanciulli and G. Scarel eds. (Springer Topics in Applied Physics, Berlin 2006); V. Fiorentini, P. Delugas, and A. Filippetti, in Advanced Gate Stacks on High-Mobility Semiconductors, A. Dimoulas, E. Gusev, P. McIntyre, and M. Heyns eds. (Springer Series in Advanced Microelectronics, Berlin, 2006).

[8] P. Delugas, V. Fiorentini, A. Filippetti, and G. Pourtois, Phys. Rev. B. 75, 115126 (2007).

[9] J. D. Gale, Phil. Mag. B 73, 3 (1996); JCS Faraday Trans. 93, 629 (1997).

[10] K. Vollmayr, W. Kob, and K. Binder, Phys. Rev. B 54, 15808 (1996).

[11] S. Baroni, A. Dal Corso, S. de Gironcoli, P. Giannozzi, C. Cavazzoni, G. Ballabio, S. Scandolo, G. Chiarotti, P. Focher, A. Pasquarello, K. Laasonen, A. Trave, R. Car, N. Marzari, A. Kokalj, http://www.pwscf.org/ The GGA approximation by J. P. Perdew, J. A. Chevary, S. H. Vosko, K. A. Jackson, M. R. Pederson, D. J. Singh, and C. Fiolhais, Phys. Rev. B 46, 6671 (1992), and ultrasoft pseudopotential according to D. Vanderbilt, Phys. Rev. B 41, R7892 (1990) are used.

[12] X. Gonze, Phys. Rev. B 55, 10337 (1997); X. Gonze and C. Lee, Phys. Rev. B 55, 10355 (1997); S. Baroni, S. de Gironcoli, A. Dal Corso, and P. Giannozzi, Rev. Mod. Phys. 73, 515 (2001).

[13] A. Filippetti and N. A. Spaldin, Phys. Rev. B 68, 045111 (2003).

[14] T. Reji et al., J. Electrochem. Soc. 154 G147 (2007); S. Van Elshocht et al., ibid. 153 F219 (2006).

[15] J. Kwo et al., J. Appl. Phys. 89, 3920 (2001). 